\title{
Iterative Learning Control Based on Relaxed 2-D Systems Stability Criteria
}

\author{
Pawel Dabkowski, Krzysztof Galkowskiy, Eric Rogers, Zhonglun Cai, \\ Chris T. Freeman, and Paul L. Lewin
}

\begin{abstract}
This brief develops a new algorithm for the design of iterative learning control law algorithms in a 2-D systems setting. This algorithm enables control law design for error convergence and performance, and is actuated by process output information only. Results are also given from the experimental application to a gantry robot.
\end{abstract}

Index Terms-2-D systems design, iterative learning control, repetitive processes.

\section{INTRODUCTION}

$\mathbf{M}$ ANY industrial systems perform the same task over a finite duration. Each execution is termed a trial and once completed the system resets to the starting location and the next trial begins. Iterative learning control (ILC) [1] uses information from the previous trial to update the control input for the next one and thereby aims to improve performance from trial-to-trial. Moreover, the next trial input is typically computed during the time taken to reset between successive trials. In comparison to other forms of control, such as adaptive, ILC adjusts the control input, a signal, instead of the controller, which is a system. The general area of ILC research is very active and one starting point for the relevant literature is the survey papers [2] and [3]. A review of the literature shows that a wide range of algorithms have been developed many of which have been experimentally tested, particularly those based on a linear plant model.

ILC can be treated as a 2-D system where one direction of information is from trial-to-trial and the other is along a trial. Since the trial length is finite, ILC can therefore be treated

Manuscript received March 4, 2010; revised March 1, 2012; accepted April 16, 2012. Manuscript received in final form May 2, 2012. This work was supported in part by the Polish Budget for Science in 2012 and 2013 as research under Project 0092-IP2-2011-71 and the Grant Agency of the Czech Republic under Grant P103-12-1794. The work of P. Dabkowski was supported in part by a Scholarship from the Foundation for Polish Science (START 2012). Recommended by Associate Editor S. S. Saab.

P. Dabkowski is with the Department of Control Engineering, Faculty of Electrical Engineering, Czech Technical University, Prague 16627, Czech Republic, and also with the Institute of Physics, Faculty of Physics, Astronomy and Informatics, Nicolaus Copernicus University, Torun 87-100, Poland (e-mail: p.dabkowski@ fizyka.umk.pl).

K. Gałkowski is with the Institute of Physics, Faculty of Physics, Astronomy and Informatics, Nicolaus Copernicus University, Torun 87-100, Poland, and also with the Institute of Control and Computation Engineering, University of Zielona Góra, Zielona Góra 65-417, Poland (e-mail: k.galkowski@issi.uz.zgora.pl).

E. Rogers, Z. Cai, C. Freeman, and P. Lewin are with the Department of Electronics and Computer Science, University of Southampton, Southampton SO17 1BJ, U.K. (e-mail: etar@ecs.soton.ac.uk; zc@ecs.soton.ac.uk; cf@ecs.soton.ac.uk; pll@ecs.soton.ac.uk).

Color versions of one or more of the figures in this paper are available online at http://ieeexplore.ieee.org.

Digital Object Identifier 10.1109/TCST.2012.2198477 as a repetitive process. These processes form a distinct class of 2-D systems where information propagation in one of the two independent directions only occurs over a finite duration, see the references in [4] for background and examples. In a repetitive process a series of sweeps, termed passes, is made through a set of dynamics defined over a finite duration known as the pass or trial, length, and at the end of each pass the process resets to its original position. The output produced on any pass is termed the pass profile and the unique control problem is that the previous pass profile acts as a forcing function on, and hence contributes, to the dynamics of the next pass profile. This can result in oscillations that increase in amplitude in one or both directions of information propagation. In this brief, we use the terminology "pass" instead of "trial" to ensure conformity with the repetitive process literature.

Recently, it has been shown [5], with experimental verification on a gantry robot system, how the repetitive process setting can be used to design control laws that consider both pass-to-pass error convergence and along the pass performance by using the repetitive process setting to impose boundedinput bounded-output (BIBO) stability independent of the pass number and the pass length. This brief gives new results where strong practical stability [6] for discrete linear repetitive processes is used, which leads to reduced complexity in design, mainly due to the need to solve lower order linear matrix inequalities (LMIs). The results of experimental application to gantry robot are given and discussed.

In this brief, $M>0$ and $M<0$ are used to denote symmetric positive definite and negative definite matrices, respectively. Also the null and identity matrices with the required dimensions are denoted by 0 and $I$, respectively. Finally, $*$ denotes the transpose of a block entry in a matrix.

\section{Problem Formulation}

The state-space model of a discrete linear repetitive process [4] has the following form over $0 \leq p \leq \alpha-1, k \geq 0$ :

$$
\begin{aligned}
x_{k+1}(p+1) & =\mathbb{A} x_{k+1}(p)+\mathbb{B} u_{k+1}(p)+\mathbb{B}_{0} y_{k}(p) \\
y_{k+1}(p) & =\mathbb{C} x_{k+1}(p)+\mathbb{D} u_{k+1}(p)+\mathbb{D}_{0} y_{k}(p)
\end{aligned}
$$

where $\alpha<\infty$ denotes the number of samples along the pass and on pass $k, x_{k}(p) \in \mathbb{R}^{n}$ is the state vector, $y_{k}(p) \in \mathbb{R}^{m}$ is the pass profile vector, and $u_{k}(p) \in \mathbb{R}^{r}$ is the control input vector. The boundary conditions are $x_{k+1}(0)=d_{k+1}, k \geq$ 0 , where the entries in $d_{k+1}$ are known constants and the initial pass profile vector $y_{0}(p)=f(p)$, where the entries in $f(p)$ are known functions of $p \in[0, \alpha-1]$. The boundary conditions, in this brief, are only of this form and hence no further explicit mention of them is made. 
The systems considered are assumed to be adequately represented by a discrete linear time-invariant system described by the state-space triple $\{A, B, C\}$. In an ILC setting, the system state-space model is written as

$$
\begin{aligned}
x_{k}(p+1) & =A x_{k}(p)+B u_{k}(p) \quad 0 \leq p \leq \alpha-1 \\
y_{k}(p) & =C x_{k}(p)
\end{aligned}
$$

where on pass $k, x_{k}(p) \in \mathbb{R}^{n}$ is the state vector, $y_{k}(p) \in \mathbb{R}^{m}$ is the output vector, and $u_{k}(p) \in \mathbb{R}^{r}$ is the control input vector. With the reference trajectory denoted by $y_{\text {ref }}(p), e_{k}(p)=$ $y_{\text {ref }}(p)-y_{k}(p)$ is the error on pass $k$.

As discussed in the introduction to this brief, ILC can be treated in a 2-D systems setting where information propagation in one direction is from pass-to-pass and in the other it is along the pass. For plants modeled by a discrete linear systems statespace model [7] used the Roesser state-space model [8] to design a control law to ensure pass-to-pass error convergence, but applications will arise where it is also necessary to take account of the along the pass dynamics. For example, consider a gantry robot whose task is to collect an object from a location, place it on a moving conveyor, and then return for the next one and so on. If, for example, the object has an open top and is filled with liquid and/or is fragile in nature, then unwanted vibrations during the transfer time could have very detrimental effects.

The basic premise in ILC is to improve performance by directly adjusting the input used on each new pass, and a commonly used method is to select the input on the current pass equal to that used on the previous pass plus a corrective term. A particular choice for the corrective term is one step ahead learning, or phase-lead ILC, of the form

$$
u_{k+1}(p)=u_{k}(p)+K e_{k}(p+1) \quad k=0,1, \ldots
$$

where $K$ is the design parameter (or matrix in the multivariable case) to be designed. In the lifting approach (the survey papers [2] and [3] are one starting point for the literature), the next step is to define the super-vector $e_{k}=\left[e_{k}^{T}(0) e_{k}^{T}(1) \cdots e_{k}^{T}(\alpha-1)\right]^{T}$ and proceed to write the controlled dynamics in the form $e_{k+1}=Q e_{k}$. This approach subsumes the along the pass dynamics and assumes that any requirements on the along the pass dynamics are, if required, met by first designing a feedback control loop for the plant and then applying lifting to the resulting state-space model.

To obtain a repetitive process description of the controlled dynamics introduce, for analysis purposes only, the state vector

$$
\eta_{k+1}(p)=x_{k+1}(p-1)-x_{k}(p-1) \text {. }
$$

Hence

$$
\eta_{k+1}(p+1)=A \eta_{k+1}(p)+B K e_{k}(p)
$$

and, since $e_{k+1}(p)-e_{k}(p)=y_{k}(p)-y_{k+1}(p)$ and using (3)

$$
e_{k+1}(p)-e_{k}(p)=-C A \eta_{k+1}(p)-C B K e_{k}(p) .
$$

The ILC dynamics can now be described by the state-space model

$$
\begin{aligned}
\eta_{k+1}(p+1) & =\hat{A} \eta_{k+1}(p)+\hat{B_{0}} e_{k}(p) \\
e_{k+1}(p) & =\hat{C} \eta_{k+1}(p)+\hat{D}_{0} e_{k}(p)
\end{aligned}
$$

where

$$
\begin{aligned}
& \hat{A}=A, \quad \hat{B}_{0}=B K \\
& \hat{C}=-C A, \quad \hat{D}_{0}=I-C B K .
\end{aligned}
$$

Equation (7) is a discrete linear repetitive process statespace model of the form (1), where $\eta$ is the state vector and the ILC error $e$ the output or pass profile vector. Hence, repetitive process stability theory can be used to study the stability and convergence properties of the ILC law considered in this brief.

The stability theory for linear repetitive processes, including those described by (1) as a special case, is based on an abstract model of the dynamics in a Banach space setting, where the contribution of the previous pass profile to the current one is of the form $y_{k+1}=L_{\alpha} y_{k}, y_{k} \in E_{\alpha}$. In this description, $E_{\alpha}$ is a Banach space with norm $\|\cdot\|$ and $L_{\alpha}$ is a bounded linear operator mapping $E_{\alpha}$ into itself. For examples described by (1), $L_{\alpha}$ is the convolution operator for a discrete linear system with (state, input, output, and direct feedthrough, respectively) state-space model matrices $\left\{\mathbb{A}, \mathbb{B}_{0}, \mathbb{C}, \mathbb{D}_{0}\right\}$.

\section{Stability and Convergence Analysis}

The stability theory for linear repetitive processes with passto-pass updating described by $y_{k+1}=L_{\alpha} y_{k}$ requires that, given any initial $y_{0}$, the sequence of pass profiles $\left\{y_{k}\right\}_{k \geq 0}$ generated converges strongly to zero as $k \rightarrow \infty$. This is termed asymptotic stability in the repetitive process setting and [4] is equivalent to the existence of real scalars $M_{\alpha}>0$ and $\lambda_{\alpha} \in(0,1)$ such that $\left\|L_{\alpha}^{k}\right\| \leq M_{\alpha} \lambda_{\alpha}^{k}, \quad k \geq 0$, where $\|\cdot\|$ also denotes the induced norm. This requires that a bounded initial pass profile produces a bounded sequence of pass profiles (BIBO stability) and the necessary and sufficient condition for this property is that $r\left(L_{\alpha}\right)<1$ where $r(\cdot)$ denotes the spectral radius. For a discrete linear repetitive process described by (1) the necessary and sufficient condition, by direct application of results in [4], is $r\left(\mathbb{D}_{0}\right)<1$, that is, all eigenvalues of $\mathbb{D}_{0}$ must lie in the open unit circle in the complex plane.

Suppose that asymptotic stability holds and let $y_{\infty}(p)$, $x_{\infty}(p)$, and $u_{\infty}(p)$ denote the strong limits as $k \rightarrow \infty$ of $y_{k+1}(p), x_{k+1}(p)$, and $u_{k+1}(p)$, respectively. Then, see [4] for the details, the limit profile corresponding to asymptotic stability of an example described by (1) is, with $\mathbb{D}=0$ for ease of presentation from this point onward

$$
\begin{aligned}
x_{\infty}(p+1) & =\left(\mathbb{A}+\mathbb{B}_{0}\left(I-\mathbb{D}_{0}\right)^{-1} \mathbb{C}\right) x_{\infty}(p)+\mathbb{B} u_{\infty}(p) \\
y_{\infty}(p) & =\left(I-\mathbb{D}_{0}\right)^{-1} \mathbb{C} x_{\infty}(p) .
\end{aligned}
$$

Consider also the special case when $\mathbb{A}=-0.5, \mathbb{B}=1$, $\mathbb{B}_{0}=0.5+\gamma, \mathbb{C}=1$, and $\mathbb{D}_{0}=0$ Asymptotic stability holds for this example and (9) becomes

$$
x_{\infty}(p+1)=\gamma x_{\infty}(p)+u_{\infty}(p) .
$$

Hence, for $|\gamma| \geq 1$, this limit profile is unstable as a standard discrete linear system and this occurs even though the state matrix $\mathbb{A}$ is stable.

To prevent cases such as the above example from arising, the BIBO property is imposed for any possible value of the pass length (mathematically this can be analyzed by 


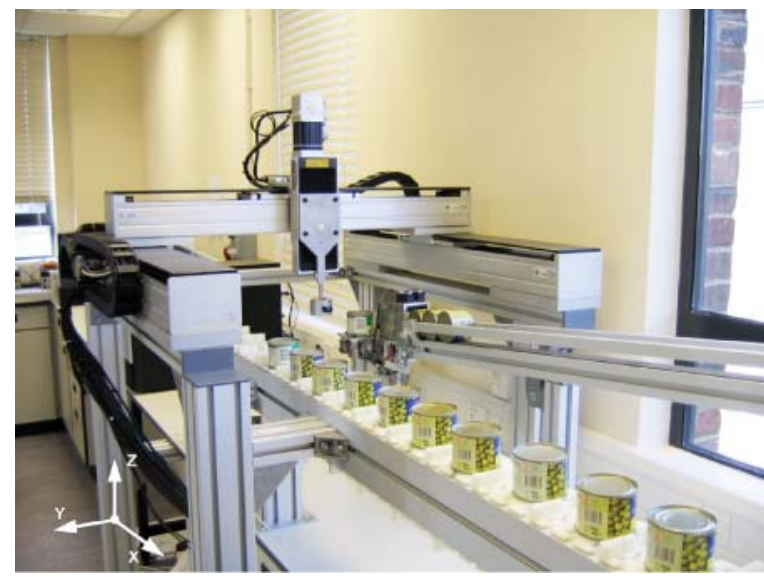

Fig. 1. Multiaxis gantry robot with the axes marked.

letting $\alpha \rightarrow \infty$ ). This is the stability along the pass property that requires the existence of finite real scalars $M_{\infty}>0$ and $\lambda_{\infty} \in(0,1)$, which are independent of $\alpha$, such that $\left\|L_{\alpha}^{k}\right\| \leq M_{\infty} \lambda_{\infty}^{k}, k \geq 0$. For the processes considered this property requires that: 1) $r\left(\mathbb{D}_{0}\right)<1$ (asymptotic stability); 2) $r(\mathbb{A})<1$; and 3 ) all eigenvalues of the transfer-function matrix $G\left(z_{1}\right)=\mathbb{C}\left(z_{1} I-\mathbb{A}\right)^{-1} \mathbb{B}_{0}+\mathbb{D}_{0}$ must lie inside the unit circle in the complex plane for all $\left|z_{1}\right|=1$. In the case of the numerical example above it is this last condition that fails.

Stability along the pass for linear repetitive processes demands that the signals involved are uniformly bounded when both independent variables $k$ and $p$ are of unbounded duration. Equivalently, this property must hold for any $k$ and $p$ in the positive quadrant of the 2-D plane, that is, $(k, p) \in P:=\{(k, p): k \geq 0, p \geq 0\}$. This requires $r\left(G\left(z_{1}\right)\right)<1$ for all $\left|z_{1}\right|=1$, which, as discussed in more detail later in this section, is a very strict condition.

Strong practical stability relaxes the BIBO stability requirement over $P$ by removing the uniform boundedness requirement as both $k \rightarrow \infty$ and $\alpha \rightarrow \infty$ but still demands this property for the cases when the pass number $k \rightarrow \infty$ and the pass length $\alpha$ finite, and also when $k$ is finite and $\alpha \rightarrow \infty$. The case when $\alpha$ is finite and $k \rightarrow \infty$ is a mathematical formulation of the requirement to operate the plant a very large number of times without the need to stop and hence, in a manufacturing example, lose throughput. The case $k$ is finite and $\alpha \rightarrow \infty$ is the mathematical formulation of the case where the process completes a finite number of passes but the pass length is very long and there is a requirement to control the along the pass dynamics.

From the analysis of asymptotic stability above, it follows that when $\alpha$ is finite and $k \rightarrow \infty$ strong practical stability requires $r\left(\mathbb{D}_{0}\right)<1$ and $r\left(\mathbb{A}+\mathbb{B}_{0}\left(I-\mathbb{D}_{0}\right)^{-1} \mathbb{C}\right)<1$. The case when $k$ is finite and $\alpha \rightarrow \infty$ results in

$$
\begin{aligned}
& y_{k+1}(\infty)=\left(\mathbb{C}(I-\mathbb{A})^{-1} \mathbb{B}_{0}+\mathbb{D}_{0}\right) y_{k}(\infty) \\
& x_{k+1}(\infty)=(I-\mathbb{A})^{-1} \mathbb{B}_{0} y_{k}(\infty)
\end{aligned}
$$

and hence we require $r\left(\mathbb{C}(I-\mathbb{A})^{-1} \mathbb{B}_{0}+\mathbb{D}_{0}\right)<1$ and $r(\mathbb{A})<1$.

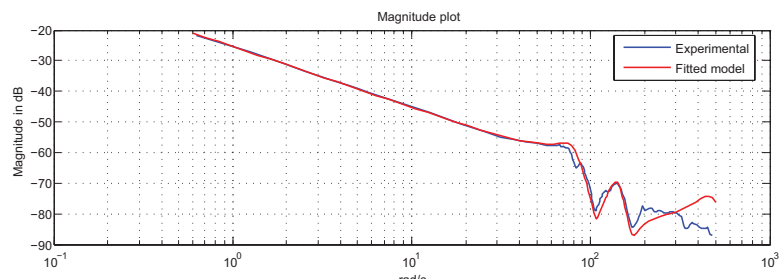

(a)

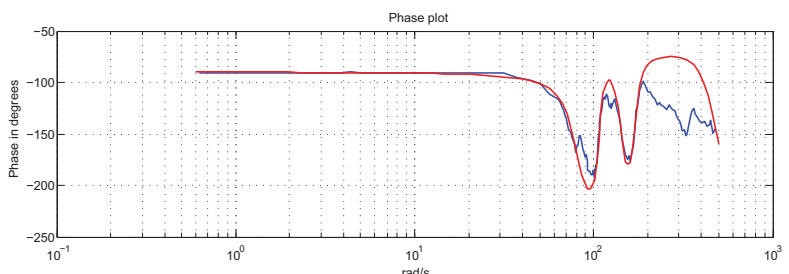

(b)

Fig. 2. (a) Bode gain and (b) phase plots for the X-axis.

In summary, therefore, strong practical stability requires that

$$
\begin{aligned}
r\left(\mathbb{D}_{0}\right) & <1 \\
r(\mathbb{A}) & <1 \\
r\left(\mathbb{A}+\mathbb{B}_{0}\left(I-\mathbb{D}_{0}\right)^{-1} \mathbb{C}\right) & <1 \\
r\left(\mathbb{C}\left(I-\mathbb{A}^{-1} \mathbb{B}_{0}+\mathbb{D}_{0}\right)\right. & <1 .
\end{aligned}
$$

Remark 1: To explain the term strong practical stability, first note that for 2-D discrete linear systems practical stability [9] was introduced in response to observations that the proposed BIBO stability was too strong for some applications. This alternative property requires that the response in each direction of information propagation is stable assuming no interaction between them. Practical stability can be extended to the processes considered here and requires conditions (12) and (13) which, as the simple example with limit profile (10) shows, is too weak in some cases.

It is also possible to characterize strong practical stability in terms of the poles of the example considered, where the concept of a pole for a 2-D linear system is much more complex than in the standard linear systems case [10]. The poles are the component-wise nonzero points in 2-D complex space where the following matrix fails to have full rank:

$$
H\left(z_{1}, z\right)=\left[\begin{array}{cc}
z_{1} I-\mathbb{A} & -\mathbb{B}_{0} \\
-\mathbb{C} & z I-\mathbb{D}_{0}
\end{array}\right] .
$$

Introducing the characteristic polynomial as

$$
\rho\left(z_{1}, z\right)=\operatorname{det} H\left(z_{1}, z\right)
$$

it follows that the poles are given by:

$$
\rho\left(z_{1}, z\right)=0 .
$$

The set $\left\{a_{1}, a_{2}\right\}$ that satisfies this last equation is termed the pole-variety. Also stability along the pass holds if and only if

$$
\rho\left(z_{1}, z\right) \neq 0, \quad\left|z_{1}\right| \geq 1 \quad|z| \geq 1 .
$$

The poles are given by the vanishing of a single 2-D nonunit polynomial and it is guaranteed to be a 1-D geometric set in 2-D complex space. Moreover, the pole variety must 


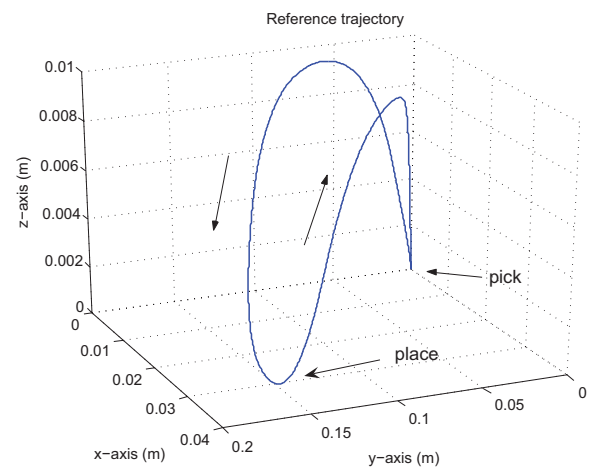

Fig. 3. 3-D reference trajectory.

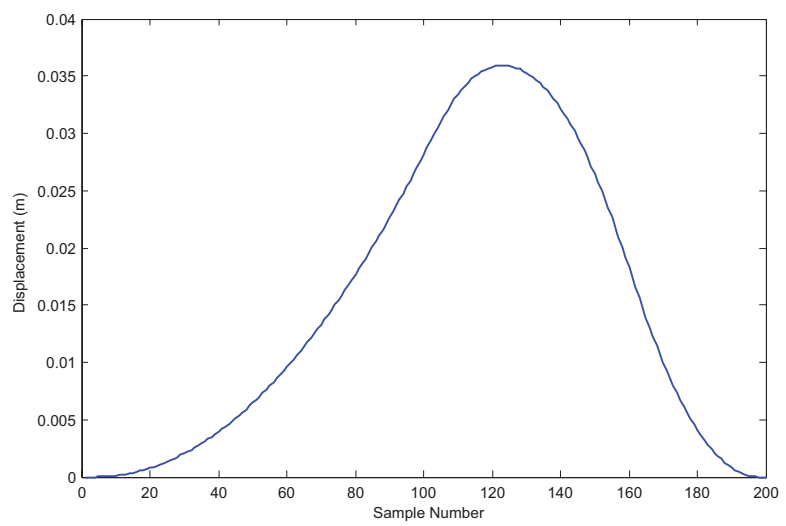

Fig. 4. $X$-axis component of the 3-D reference trajectory.

be complex, even though the entries of the matrices $\mathbb{A}, \mathbb{B}_{0}, \mathbb{C}$, and $\mathbb{D}_{0}$ are real. This property is required to capture the full exponential-type dynamics of the process.

The poles of discrete linear repetitive processes can be interpreted in terms of exponential trajectories, which in the case considered here have a clear physical interpretation. In particular, stability along the pass requires no poles with $\left|z_{1}\right| \geq 1$ and $|z| \geq 1$, which is a direct generalization of the standard linear systems case. For strong practical stability, it is easy to see that (14) is equivalent to $\rho\left(z_{1}, 1\right) \neq 0,\left|z_{1}\right| \geq 1$, and (15) to $\rho(1, z) \neq 0,|z| \geq 1$. Hence for this stability property, the only exponential trajectories considered are identical to those for a standard discrete linear system and these are clearly a subset of those given by (18).

In terms of design to track a given reference vector, imposing the requirement for stability along the pass means that the control law must achieve the required level of attenuation over the complete frequency range [due to condition 3) above expressed in terms of the transfer-function matrix $\left.G\left(z_{1}\right)\right]$ and, by comparison with the standard linear systems case, this is most likely to result in a very difficult design problem. In such cases, strong practical stability may lead to acceptable design, especially for applications where an unstable limit profile is not acceptable and/or some control is required over the along the pass dynamics.

The following result is the basis for the ILC design developed and experimentally tested in this brief.

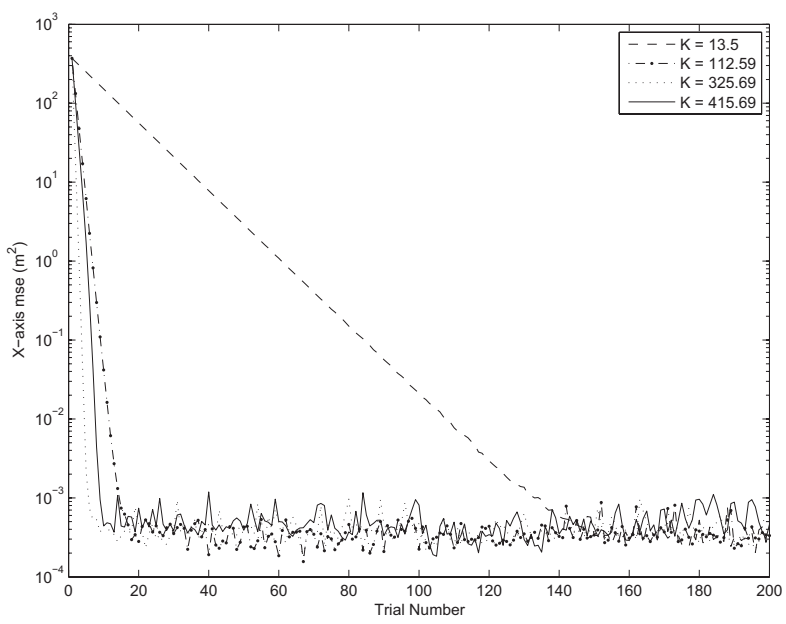

Fig. 5. MSE against pass number $(k)$ when $\hat{K}_{1}=-45.5$ is constant for different $K$ shown in the legend.

Theorem 1 [6]: A discrete linear repetitive process described by (1) is strongly practically stable if and only if there exist matrices $W_{1}>0, W_{2}>0, Q_{1}>0, Q_{2}>0$, and nonsingular matrices $S_{1}$ and $S_{2}$ such that the following set of LMIs is feasible:

$$
\begin{aligned}
& {\left[\begin{array}{cc}
-W_{1} & W_{1} \mathbb{D}_{0}^{T} \\
\mathbb{D}_{0} W_{1} & -W_{1}
\end{array}\right]<0} \\
& {\left[\begin{array}{ll}
-W_{2} & W_{2} \mathbb{A}^{T} \\
\mathbb{A} W_{2} & -W_{2}
\end{array}\right]<0} \\
& {\left[\begin{array}{cc}
-Q_{1} & S_{1}^{T} \mathbb{A}_{1}^{T} \\
\mathbb{A}_{1} S_{1} & Q_{1}-\mathbb{E}_{1} S_{1}-S_{1}^{T} \mathbb{E}_{1}^{T}
\end{array}\right]<0} \\
& {\left[\begin{array}{cc}
-Q_{2} & S_{2}^{T} \mathbb{A}_{2}^{T} \\
\mathbb{A}_{2} S_{2} & Q_{2}-\mathbb{E}_{2} S_{2}-S_{2}^{T} \mathbb{E}_{2}^{T}
\end{array}\right]<0}
\end{aligned}
$$

where

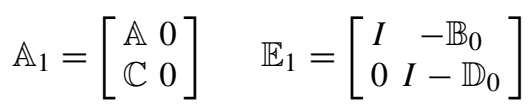

and

$$
\mathbb{A}_{2}=\left[\begin{array}{ll}
0 & \mathbb{B}_{0} \\
0 & \mathbb{D}_{0}
\end{array}\right] \quad \mathbb{E}_{2}=\left[\begin{array}{cc}
I-\mathbb{A} & 0 \\
-\mathbb{C} & I
\end{array}\right] .
$$

Consider the application of the repetitive process stability analysis to the ILC state-space model described by (7) and (8). The first result is the following.

Lemma 1 [11]: The ILC state-space model described by (7) and (8) cannot be strongly practically stable if the matrix CBK is singular. If the matrix CBK is nonsingular, the condition (14) always holds.

Remark 2: In previous work, such as [5] ILC design using stability along the pass for discrete linear repetitive processes was considered. The results obtained are also expressed in terms of LMIs which are sufficient, but not necessary, for the stability property. In contrast, the conditions for strong practical stability are necessary and sufficient. Strong practical stability is a necessary condition for stability along the pass, but the conditions under which strong practical stability hold cannot be obtained as special cases of those for stability along the pass. The reason for this is, as discussed above, that stability along the pass and, in particular, the poles are 
defined in terms of two indeterminates whereas for strong practical stability they are defined in standard discrete linear systems terms. The control law in [5] also used the current pass state vector. Previous work has shown how to replace current pass state feedback with pass profile (output) based control action [11] and design for strong practical stability.

\section{ILC DESIGN}

Using Lemma 1, the requirement that (14) holds in the ILC application is redundant and the following are now an obvious set of necessary and sufficient conditions for strong practical stability.

Theorem 2: The ILC state-space model given by (7) and (8) with CBK nonsingular is strongly practically stable if and only if

$$
\begin{aligned}
r(I-\mathrm{CBK}) & <1 \\
r(A) & <1 \\
r\left(I-\mathrm{CBK}-\mathrm{CA}(I-A)^{-1} \mathrm{BK}\right) & <1 .
\end{aligned}
$$

Condition (24) is that obtained from applying the result in [7] obtained by a 2-D Roesser model analysis with the control law (3). To illustrate the problems that can result if (26) does not hold, consider the case of (2) with the ILC law applied when $A=0.9$ and $\mathrm{CBK}=1 / 2$. Then (24) and (25) hold but not (26) and, by (11), unacceptable passto-pass dynamics will result. Hence in ILC applications, it is possible that stable plants will produce unacceptable dynamics and a design method that prevents such cases from arising, by enforcing both (25) and (26), is required. The new design algorithm of this brief meets this requirement and is a major advantage of the 2-D systems approach.

The aim is to develop a computationally efficient method of designing the control law. The task is more complicated than that of deriving a test for strong practical stability since there is only one matrix $K$ that has to simultaneously satisfy the first and third conditions of Theorem 2 .

Introduce the notation

$$
\begin{aligned}
A_{2} & =\left[\begin{array}{ll}
0 & \mathrm{BK} \\
0 & I-\mathrm{CBK}
\end{array}\right]=\widetilde{A}_{2}+\widetilde{\Pi} \widetilde{K} \\
E_{2} & =\left[\begin{array}{cc}
I-A & 0 \\
C A & I
\end{array}\right]
\end{aligned}
$$

where

$$
\widetilde{A}_{2}=\left[\begin{array}{ll}
0 & 0 \\
0 & I
\end{array}\right], \quad \widetilde{\Pi}=\left[\begin{array}{c}
B \\
-C B
\end{array}\right], \quad \widetilde{K}=\left[\begin{array}{ll}
0 & K
\end{array}\right] .
$$

The following is the major new result of this brief.

Theorem 3: The ILC scheme described by (7) with $r(A)<1$ and $\mathrm{CBK}$ nonsingular is strongly practically stable if

$$
\left[\begin{array}{cc}
-Q_{2} & S^{T} \widetilde{A}_{2}^{T}+\widetilde{N}^{T} \widetilde{\Pi}^{T} \\
\widetilde{A}_{2} S+\widetilde{\Pi} \widetilde{N} & Q_{2}-E_{2} S-\left(E_{2} S\right)^{T}
\end{array}\right]<0
$$

holds for $Q_{2}>0$, nonsingular matrix $S=\operatorname{diag}\left(S_{1}, S_{2}\right)$ and rectangular matrix $\widetilde{N}=[0 N]$. Also if (28) holds, a stabilizing $K$ is given by

$$
K=N S_{2}^{-1} .
$$
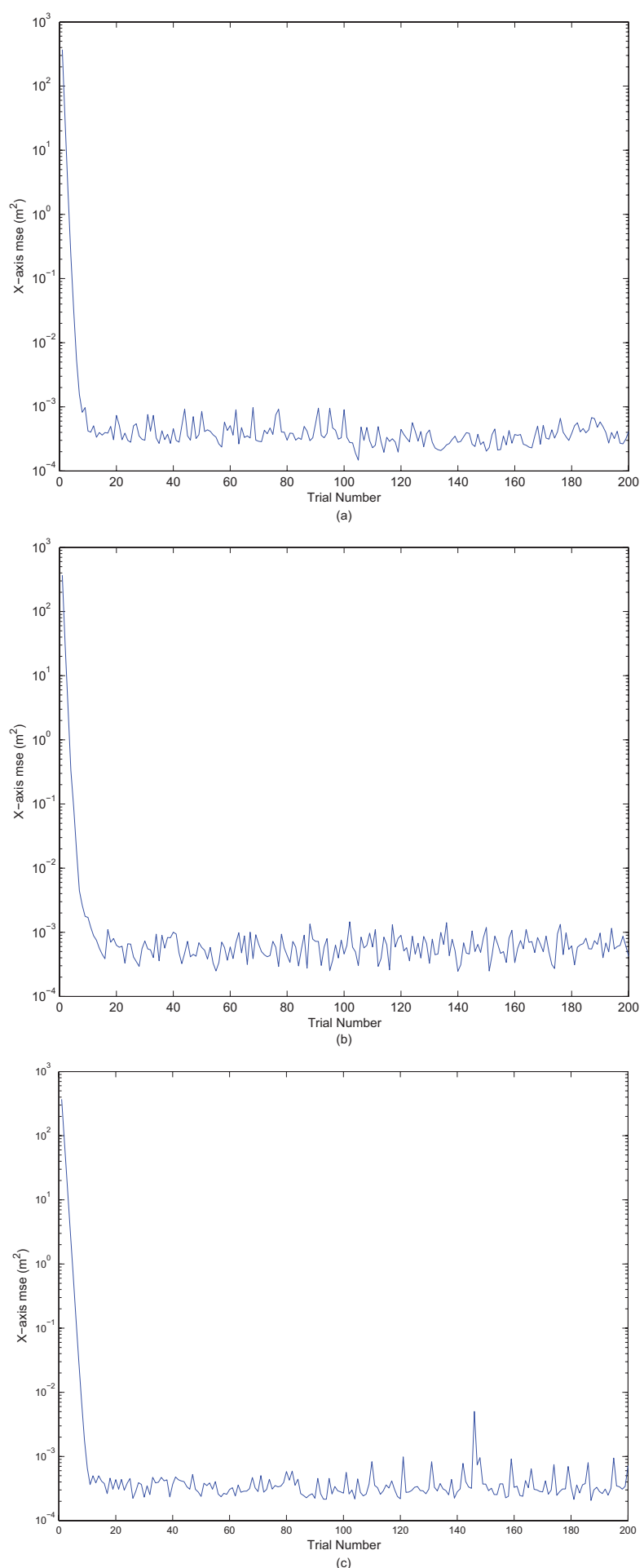

Fig. 6. MSE against pass number $(k)$ for three values of $\hat{K}_{1}$ and constant value of $K=190$. (a) MSE against pass number $(k)$ for $\hat{K}_{1}=-257.60$ and $K=190$. (b) MSE against pass number $(k)$ for $\hat{K}_{1}=-157.47$ and $K=190$. (c) MSE against pass number $(k)$ for $\hat{K}_{1}=-357$ and $K=190$.

Proof: First, (28) guarantees that (26) of Theorem 2 holds. This follows by applying the fourth condition of Theorem 1 to the LMI (28) with the previously introduced notation. To find the stabilizing control law matrix $K$, we have to use the block diagonal matrix $S=\operatorname{diag}\left(S_{1}, S_{2}\right)$ and introducing the additional variable $N=K S_{2}$ completes this part of the proof. 
The next step is to show that the LMI (28) guarantees that (24) holds, where this latter condition is equivalent to following LMI:

$$
\left[\begin{array}{cc}
-W_{1} & S_{2}^{T}-N^{T} B^{T} C^{T} \\
S_{2}-C B N & W_{1}-S_{2}-S_{2}^{T}
\end{array}\right]<0
$$

and hence (28) can be written in the following extended form using the matrices defined in (27):

$$
\left[\begin{array}{cccr}
-Q_{211} & * & * & * \\
-Q_{212}^{T} & -Q_{222} & * & * \\
0 & \mathrm{BN} & \Upsilon & * \\
0 & S_{2}-\mathrm{CBN} & Q_{212}^{T}-\mathrm{CAS}_{1} \Omega
\end{array}\right]<0
$$

where $\Upsilon=Q_{211}-S_{1}-S_{1}^{T}+A S_{1}+S_{1}^{T} A^{T}$ and $\Omega=Q_{222}-S_{2}-S_{2}^{T}$. For (31) to hold the following must be satisfied [the lower $3 \times 3$ block principal minor of the matrix in (31)]:

$$
\left[\begin{array}{cc|c}
-Q_{222} & N^{T} B^{T} & S_{2}^{T}-N^{T} B^{T} C^{T} \\
\mathrm{BN} & \Upsilon & Q_{212}-S_{1}^{T} A^{T} C^{T} \\
\hline S_{2}-\mathrm{CBN} & Q_{212}^{T}-\mathrm{CAS}_{1} & \Omega
\end{array}\right]<0 .
$$

Next, left- and right-multiply this last result by

$$
\left[\begin{array}{ll}
0 & I \\
I & 0
\end{array}\right]
$$

to obtain

$$
\left[\begin{array}{c|cc}
\Omega & S_{2}-C B N & Q_{212}^{T}-C A S_{1} \\
\hline\left(S_{2}-\mathrm{CBN}\right)^{T} & -Q_{222} & N^{T} B^{T} \\
\left(Q_{212}^{T}-\mathrm{CAS}_{1}\right)^{T} & \mathrm{BN} & \Upsilon
\end{array}\right]<0 .
$$

For (33), with $\Omega$ defined by (31), to hold

$$
\left[\begin{array}{cc}
Q_{222}-S_{2}-S_{2}^{T} & S_{2}-\mathrm{CBN} \\
\left(S_{2}-\mathrm{CBN}\right)^{T} & -Q_{222}
\end{array}\right]<0
$$

Finally, left- and right-multiply this last inequality by

$$
\left[\begin{array}{ll}
0 & I \\
I & 0
\end{array}\right]
$$

to obtain (30) with $Q_{222}=W_{1}$ and the proof is complete.

The result of this theorem is sufficient but not necessary since there is only one matrix $K$ available for selection to simultaneously satisfy two conditions. This, of course, results in possible conservativeness but note again that in design for stability along the pass both the stability condition and the control law design are based on sufficient only conditions whereas with strong practical stability only the latter requirement has this property.

Theorem 3 requires that the plant matrix $A$ is stable. If this is not the case, apply the preliminary control law

$$
u_{k}(p)=\hat{K}_{1} y_{k}(p)=\hat{K}_{1} C x_{k}(p)
$$

which is output based to avoid the need for state vector measurements or the use of a state observer. The following lemma is an immediate consequence of the main result in [12].

Lemma 2 [12]: Suppose that the control law (35) is applied to (2). Then $r\left(A+B \hat{K}_{1} C\right)<1$ in the resulting state-space

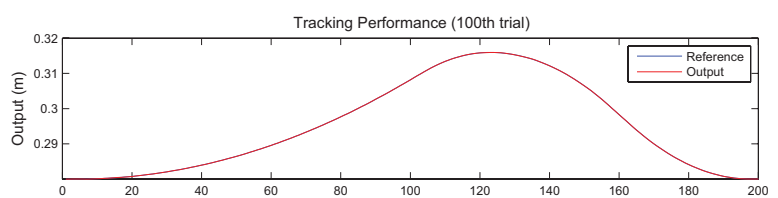

(a)

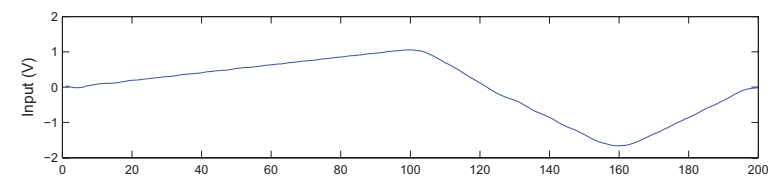

(b)

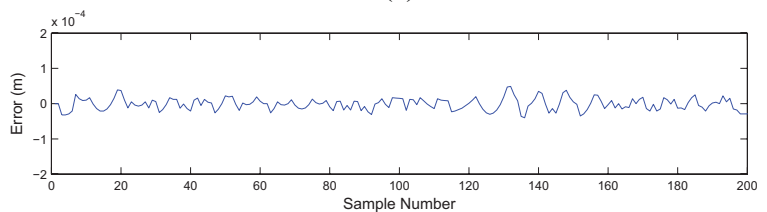

(c)

Fig. 7. (a) Output, (b) input, and (c) error for pass $k=100$ when $\hat{K}_{1}=$ -300.29 and $K=325.69$.

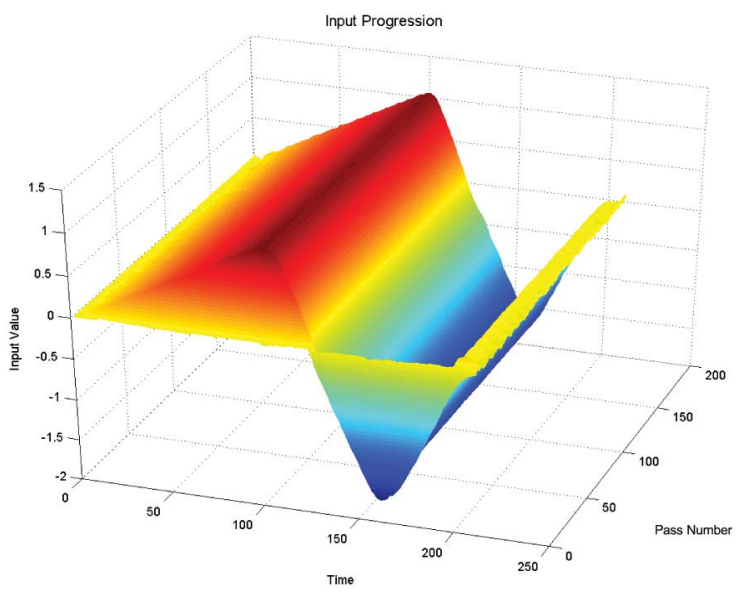

Fig. 8. Input progression for $\hat{K}_{1}=-300.29$ and $K=325.69$.

model if there exist matrices $V>0, F, M$, and $S$ such that the following LMI holds:

$$
\begin{gathered}
{\left[\begin{array}{cc}
-V & (\mathrm{AS}+\mathrm{BFC})^{T} \\
A S+\mathrm{BFC} & V-S-S^{T}
\end{array}\right]<0} \\
\mathrm{MC}=\mathrm{CS} .
\end{gathered}
$$

If this LMI holds, stabilizing $\hat{K}_{1}$ can be computed using

$$
\hat{K}_{1}=F M^{-1} \text {. }
$$

The control law applied to the plant is

$$
\begin{aligned}
u_{k+1}(p)= & u_{k}(p)+\hat{K}_{1}\left[y_{k+1}(p)-y_{k}(p)\right] \\
& +K\left[y_{\mathrm{ref}}(p+1)-y_{k}(p+1)\right]
\end{aligned}
$$

where the matrices $\hat{K}_{1}$ and $K$ are obtained using Lemma 2 and Theorem 3 as appropriate. The control law (39) (see, for example, [13]) is known in the ILC literature and what is developed in this brief is a new design method. This method automatically enforces (26) and in comparison to alternative designs this is a major advantage of 2-D systemsbased analysis and control law design. 


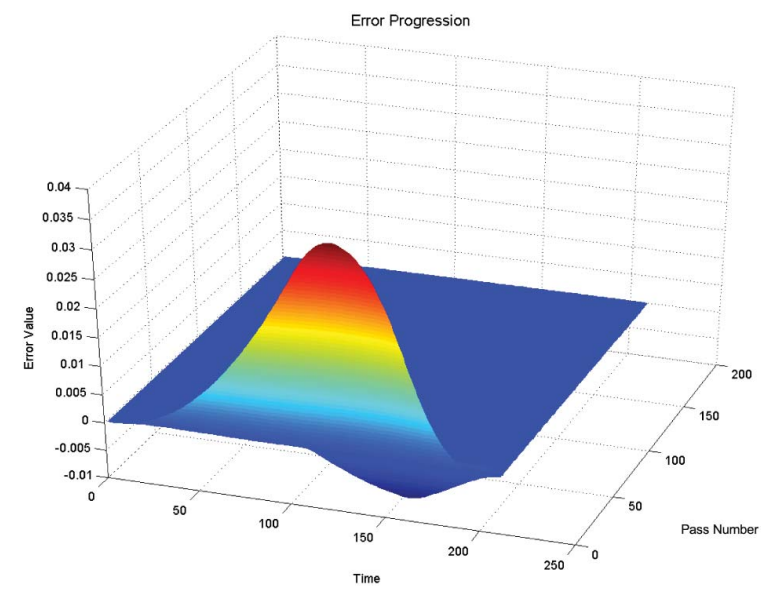

Fig. 9. Error progression for $\hat{K}_{1}=-300.29$ and $K=325.69$.

\section{EXPERIMENTAL VERIFICATION}

To experimentally assess the new design method, it was applied to a multiaxis gantry robot. This robot, shown in Fig. 1 with the axes marked, has previously been used for testing and comparing the performance of other ILC algorithms, see, for example, [14]. Each axis of the gantry robot is modeled based on frequency response tests where, since the axes are orthogonal, it is assumed that there is minimal interaction between them. An approximate continuous-time transfer-function representation for each of the three axes has been obtained through use of straight line approximations of the Bode gain plots. Here, we only give the final result for the $X$-axis in the form of a seventh-order transfer-function and those for the $y$ and $z$-axis can be found in [14] and [15], including a detailed description of how the transfer-function used for design was obtained from the Bode plots.

The Bode gain and phase plots for the $X$-axis are shown in Fig. 2, and the resulting transfer-function used for design is shown in (40) at the bottom of the page.

This robot system is designed to emulate a pick and place task that arises in many applications to which ILC is applicable. In operation, the robot must undertake the following operations in synchronization with a conveyor system: 1) collect an object from a fixed location; 2) transfer it over a finite duration; 3) place it on the moving conveyor; 4) return to the original location for the next object; and 5) repeat the previous four steps for as many objects as required. The 3-D trajectory used in the results given here is shown in Fig. 3 with the $X$-axis component in Fig. 4.

The model used for the extensive experimental investigation, of which the results given in this section are a representative selection, was constructed using zero-order hold discretization with sampling period of $0.01 \mathrm{~s}$.

In the control law (39), the $\hat{K}_{1}$ term is required and use of Lemma 2 gives one choice as $\hat{K}_{1}=-45.5$. In the first set of experiments, the trends resulting from particular strategies for selecting $\hat{K}_{1}$ and $K$ in (39) were investigated. The first case considered was with constant $\hat{K}_{1}$, that is, the eigenvalues

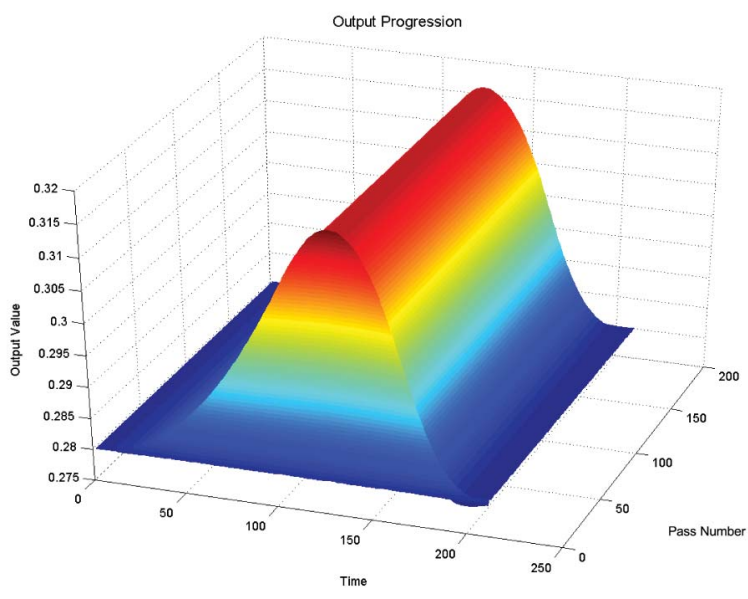

Fig. 10. Output progression for $\hat{K}_{1}=-300.29$ and $K=325.69$.

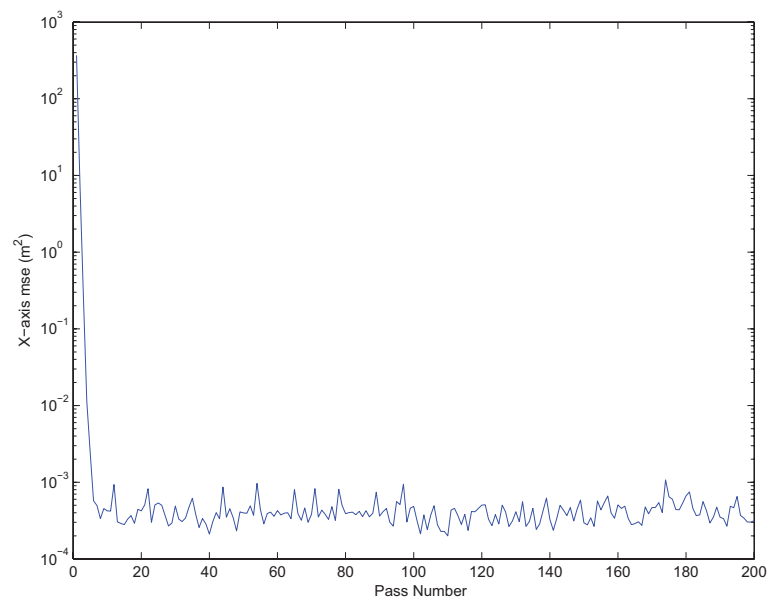

Fig. 11. MSE against pass number $(k)$ for $\hat{K}_{1}=-300.29$ and $K=325.69$.

of the matrix $A$ are fixed once $\hat{K}_{1}$ is selected, and the design task is then to chose a corresponding $K$, where the LMIs to be solved provide a family of solutions.

Consider the case when $\hat{K}_{1}=-45.5$. Then admissible values of $K$ include 13.5, 112.59, 325.69, 415.69, and Fig. 5 shows the mean squared error (MSE) for these choices plotted on one axis. These indicate that increasing the value of $K$ leads to faster convergence from pass-topass but also that performance begins to degrade again if $K$ is too large. This demonstrates that enforcing fast pass-topass error convergence can result in degraded along the pass performance.

To consider the effects of focusing on the selection of $\hat{K}_{1}$, consider the case when $K=190$ and $\hat{K}_{1}=-257.60,-157.47$, and -357 , respectively. Inspecting the results of Fig. 6 confirms that the speed of the pass-topass error convergence is unchanged (as expected with the fixed $K$ ), but the levels of oscillation in the error vary and hence the possibility of potentially large-scale oscillations in the along the pass responses.

$$
G_{X}(s)=\frac{13077183.4436(s+113.4)}{s\left(s^{2}+61.57 s+1.125 \times 10^{4}\right)} \times \frac{\left(s^{2}+30.28 s+2.13 \times 10^{4}\right)}{\left(s^{2}+227.9 s+5.647 \times 10^{4}\right)\left(s^{2}+466.1 s+6.142 \times 10^{5}\right)}
$$


Since in applications only a finite number of trials will ever be completed, clearly there is interest in selecting $\hat{K}_{1}$ and $K$ to give the desired level of performance in terms of, for example, the minimum MSE over a finite number of trials, say 200. On the basis of extensive numerical evaluations the case is considered when $\hat{K}_{1}=-300.29$ and $K=325.69$, where Fig. 7 shows the experimentally measured output, input and error on pass $k=100$ and Figs. 8-10 the progression with pass number $k$ of the input, error, and output, respectively. Finally, Fig. 11 shows the resulting MSE against pass number $k$.

\section{CONCLUSION}

This brief has continued the development of design algorithms for ILC schemes using a 2-D system setting for analysis, where both pass-to-pass error convergence and along the trial performance were considered. The control law is phase-lead ILC augmented by output feedback on the current pass if required. This form of control law is well known in the literature where if the output feedback term $\left[\hat{K}_{1}\right.$ in (35)] is required to pre-stabilize the plant dynamics and also meet any performance specifications on the along the trial dynamics), many design methods exist. These were based on first selecting $\hat{K}_{1}$ and then applying ILC via, for example, lifting to the resulting controlled plant. It is, however, possible that placement of the poles of the plant in stable locations will not guarantee acceptable along the pass dynamics as consideration of the limiting case when $K$ is finite and the pass length $\alpha \rightarrow \infty$ via (11) and hence Theorem 1 shows. If such a case arises in an application, the algorithm in this brief automatically enforces (26) for the $\hat{K}_{1}$ arising from the stabilization of the plant matrix. The LMI-based design also produces a family of solutions and hence permits the opportunity to tune the final design. Furthermore, the LMIs are of lower dimension and potentially less conservative than alternatives from the repetitive process-based approach to ILC design. In more general terms, the 2-D systems approach does allow simultaneous design for pass-to-pass error convergence and also transient response along the passes. The latter aspect requires the ability to arbitrarily assign the spectrum of the state matrix and if this is not possible, as with the particular control law in this brief where (35) is a static output feedback for the plant, then the control law structure must be enhanced.

The ILC law design algorithm developed in this brief has been experimentally tested on a gantry robot that has been previously used to benchmark other ILC algorithms. Hence, the same reference signal has been used. The experimental results show that the design method of this brief is capable of delivering high quality performance in terms of pass-to-pass error convergence and along the pass performance. Much further work is required before a detailed comparative assessment of this design method against alternatives can be undertaken. One immediate issue is robust control to allow for uncertainty in the model to be used for design. Also there is a need to do more comparative experimental studies to fully determine the merits of this algorithm against alternatives.

\section{REFERENCES}

[1] S. Arimoto, S. Kawamura, and F. Miyazaki, "Bettering operations of robots by learning," J. Robot. Syst., vol. 1, no. 2, pp. 123-140, 1984.

[2] H.-S. Ahn, Y. Chen, and K. L. Moore, "Iterative learning control: Brief survey and categorization," IEEE Trans. Syst. Man Cybern. C, vol. 37, no. 6, pp. 1099-1121, Nov. 2007.

[3] D. A. Bristow, M. Tharayil, and A. Alleyne, "A survey of iterative learning control," IEEE Control Syst. Mag., vol. 26, no. 3, pp. 96-114, Jun. 2006.

[4] E. Rogers, K. Gałkowski, and D. H. Owens, Control Systems Theory and Applications for Linear Repetitive Processes (Lecture Notes in Control and Information Sciences). Berlin, Germany: Springer-Verlag, 2007.

[5] L. Hładowski, K. Gałkowski, Z. Cai, E. Rogers, C. T. Freeman, and P. L. Lewin, "Experimentally supported 2-D systems based iterative learning control law design for error convergence and performance," Control Eng. Pract., vol. 18, no. 4, pp. 339-348, Apr. 2010.

[6] P. Dabkowski, K. Galkowski, O. Bachelier, E. Rogers, and J. Lam, "A new approach to strong practical stability and stabilization of discrete linear repetitive processes," in Proc. 19th Int. Symp. Math. Theory Netw. Syst., Budapest, Hungary, 2009, pp. 311-317.

[7] J. E. Kurek and M. B. Zaremba, "Iterative learnig control synthesis based on 2-D system theory," IEEE Trans. Autom. Control, vol. 38, no. 1, pp. 121-125, Jan. 1993.

[8] R. P. Roesser, "A discrete state-space model for linear image processing," IEEE Trans. Autom. Control, vol. 20, no. 1, pp. 1-10, Feb. 1975.

[9] P. Agathoklis and L. T. Bruton, "Practical-BIBO stability of ndimensional discrete systems," IEEE Proc. G, Electron. Circuits Syst., vol. 130, no. 6, pp. 236-242, Dec. 1983.

[10] J. Wood, U. Oberst, E. Rogers, and D. H. Owens, "A behavioural approach to the pole structure of one-dimensional and multidimensional linear systems," SIAM J. Control Optim., vol. 38, no. 2, pp. 627-61, Jan. 2000

[11] P. Dabkowski, K. Gałkowski, E. Rogers, Z. Cai, C. T. Freeman, and P. L. Lewin, "Iterative learning control based on strong practical stability of repetitive processes," in Proc. 48th IEEE Conf. Decision Control, Dec. 2009, pp. 4864-4869.

[12] A. Trofino, "Parameter-dependent Lyapunov functions for a class of uncertain linear systems: An LMI approach," in Proc. 38th IEEE Conf. Dec. Control, Dec. 1999, pp. 2341-2346.

[13] A. Tayebi and M. B. Zaremba, "Iterative learning control design is straightforward for uncertain LTI systems satisfying the robust performance condition," IEEE Trans. Autom. Control, vol. 48, no. 1, pp. 101106, Jan. 2003.

[14] J. D. Ratcliffe, P. L. Lewin, E. Rogers, J. J. Hatonen, and D. H. Owens, "Norm-optimal iterative learning control applied to gantry robots for automation applications," IEEE Trans. Robot., vol. 22, no. 6, pp. 13031307, Dec. 2006.

[15] J. D. Ratcliffe, "Iterative learning control implemented on a multi-axis system," Ph.D. thesis, Dept. Electron. Comput. Sci., Univ. Southampton, Southampton, U.K., 2005. 